\title{
Effects of Polarization on a Single-mode Class A Laser
}

\author{
Fumiyoshi KUWASHIMA, ${ }^{1}$ and Hiroshi IWASAWA ${ }^{2}$ \\ ${ }^{1}$ Kagoshima National College of Technology,1460-1, Sinkou Hayato-cho, Kirishima-shi, Kagoshima, 899-5193
}

${ }^{2}$ Fukui University, Takasu, 3-3-40-304 Nishi-ku, Hiroshoma 733-0871

(Received January 29, 2008)

\begin{abstract}
Effects of the light polarization on a chaotic oscillation dynamics of a single-mode $\mathrm{He}-\mathrm{Ne}(6326 \AA)$ class A laser was studied by using optical delayed feedback with an external optical resonator. We observed time series, spectra, phase portraits and Lyapunov exponents for each linear and random polarization lasers.
\end{abstract}

Key Words: Class A laser, Chaos, Polarization, Lyapunov exponents

\section{Introduction}

In the past three decades, the onset of chaos in dissipative dynamical systems has been extensively explored in a large variety of physical systems. The minimal condition for the onset of the deterministic chaos in a dissipative system with continuous variables is the presence of at least three degree of freedom, which was proved by Ruelle and Takens ${ }^{1)}$.

The single mode laser is a typical dissipative dynamical system with three degree of freedom under the specific conditions. The onset of chaos and its conditions for a laser were first predicted theoretically by Haken, in which the equivalence of the single-mode homogeneous broadening laser equations to Lorenz equations and threshold value of the chaos under the bad cavity condition were derived. ${ }^{2)}$ However, it is difficult to realize this condition for practical lasers. In 1982, chaotic emission from a $3.39 \mu \mathrm{m}$ He-Ne laser was first observed by Weiss and King ${ }^{3)}$ under the bad cavity condition. After this experimental, many experiments on the chaos of several kind of lasers have been rapidly pursued as reviewed in Refs. 4 and 5 .

With regard to laser dynamics, lasers are classified into class $\mathrm{A}, \mathrm{B}$, and $\mathrm{C}$ on the basis of their relationship between decay rates of three relevant variables electric field, population inversion and polarization, which are given by the relaxation rates $\kappa, \gamma_{\|}$and $\gamma_{\perp}$, respectively ${ }^{6}$. The dynamical behavior of the single mode laser is described by three equations for these three relevant variables that usually decay on very different time scales.

Most experimental and theoretical investigations on chaotic dynamics for lasers have been carried out for class B and class $\mathrm{C}$ lasers.

Lasers whose population inversion and polarization decay faster than their electric fields are called class A lasers. The laser equations for a class A laser can be reduced to one that is for only the field. In order to generate chaos in this class lasers, at least two external forces must be added. To our knowledge, not many experimental studies of class A lasers have been reported. Although chaotic types of behavior in a four-mode $\mathrm{He}-\mathrm{Ne}(6328 \AA)$ standing wave laser and a gas ring laser were studied by Chetverikov ${ }^{7}$ and Danileiko ${ }^{8)}$ et al., respectively, they did not study the single-mode laser. Furthermore, they observed only time behavior and spectra and did not confirm chaos from the Lyapunov exponents. Recently, as reported in our previous papers, the chaotic oscillation of a single mode class A standing wave laser has been observed by optical delayed feedback using an external resonator ${ }^{9-11)}$. The chaotic dynamics of a single-mode class A laser with optical delayed feedback has also been investigated theoretically ${ }^{12}$. In that paper, we derived the equation for the single-mode class A laser equation and using this model, the characteristic frequency is deduced as the round-trip frequency. The characteristic frequency of a single mode class A laser with optical delayed feedback was investigated by using a long external resonator ${ }^{13)}$. In that paper, although the round trip frequency was observed but the spectrum is not broadened around the round trip frequency and it is broadened at approximately $10-100 \mathrm{~Hz}$. This result is not inconsistent with theoretical one.

Considering these situation, effects of linear and random polarization on chaotic dynamics of a single-mode $\mathrm{He}-\mathrm{Ne}$ $(6328 \AA)$ class A laser are investigated by optical delayed feedback with an external optical resonator. In our previous papers, it is found that the fundamental periodicity is lower region $(10-100 \mathrm{~Hz})$ and the fluctuation of the population inversion may be one of origin of this phenomenon. In this proceeding, we investigate the effect of light polarizations in order to estimate the dynamical behavior of the class A lasers.

We observe time series, spectra, phase portraits and Lyapunov exponents for linear and random polarized lasers.

\section{Experimental Setup}

The experimental setup of our system is shown in Fig.1.

We use two He-Ne lasers. One is a random polarization laser (Sigma-Koki 05-LHR-211), and the other is a linear polarization laser (Sigma-Koki 05-LHP-211). Using Brewster windows, a linear polarization laser is realized. The light polarization is changed by exchange of laser tubes.

A single-mode $\mathrm{He}-\mathrm{Ne}(6328 \AA)$ laser was operated in transverse $\left(\mathrm{TEM}_{00}\right)$ and longitudinal single mode at a frequency interval of $1.219 \mathrm{GHz}$ between the longitudinal modes of the resonator which is similar to the laser medium's gain width of $1.5 \mathrm{GHz}$. The resonator consists of two mirrors $\mathrm{M}_{1}$ and $\mathrm{M}_{2}$ with reflectivity's (power) $\mathrm{R}_{1}=100 \%$ and $\mathrm{R}_{2}=99 \%$, respectively. Hence, this is a very good cavity. 


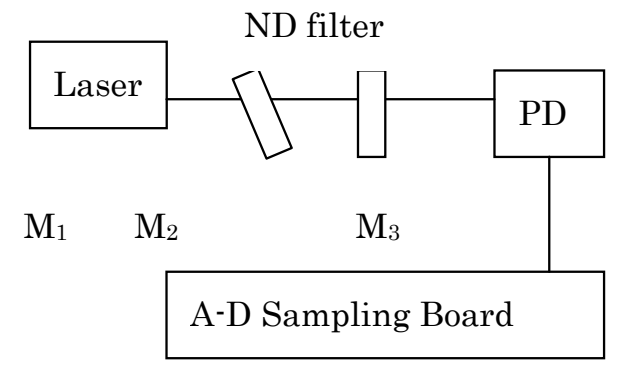

Fig.1. Experimental setup; Laser is $\mathrm{He}-\mathrm{Ne}$ laser of random polarization or linear polarization.

The single-mode He-Ne laser $(6328 \AA)$ is a typical class A laser, because the lifetime of a photon $\left(\tau_{\mathrm{p}}=10^{-5} \mathrm{~s}\right)$ is much longer than the transverse and longitudinal relaxation times $\left(\mathrm{T}_{2}=1 / \gamma_{\perp}=10^{-8} \mathrm{~s}, \mathrm{~T}_{1}=1 / \gamma_{\|}=10^{-7} \mathrm{~s}\right)$. That is, the laser operates as a one degree of freedom dissipative system. So, the output power is fed back into the He-Ne lasers via the external mirror $\mathrm{M}_{3}$, and the output signal from $\mathrm{M}_{3}$ is detected using a photodiode with a response time of $1 \mathrm{~ns}$.

Feedback rates are adjusted by a neutral-density (ND) filter; the effective reflectivity of the external mirror $\mathrm{M}_{3}$ is denoted by $R_{(3 e f f)}=T^{2} R_{3}$, where $T$ is the transmission coefficient of the ND filter.

The length of the laser inner resonator is about $12.3 \mathrm{~cm}$. The length of the external resonator $\mathrm{L}_{2}$ is $12.3 \mathrm{~cm}$, which is similar to that of inner resonator. The output power is about $0.84 \mathrm{~mW}$ under $\mathrm{CW}$ operation at a fixed discharge current of $4 \mathrm{~mA}$.

\section{Experimental Results and Discussion}

In this experiment, the control parameters are delayed feedback rate and light polarizations.

The time sequences of the output power from the external mirror are shown in Figs. 2(a)-2(f), in which the length of external mirror is $12.3 \mathrm{~cm}$, which is the same as that of the

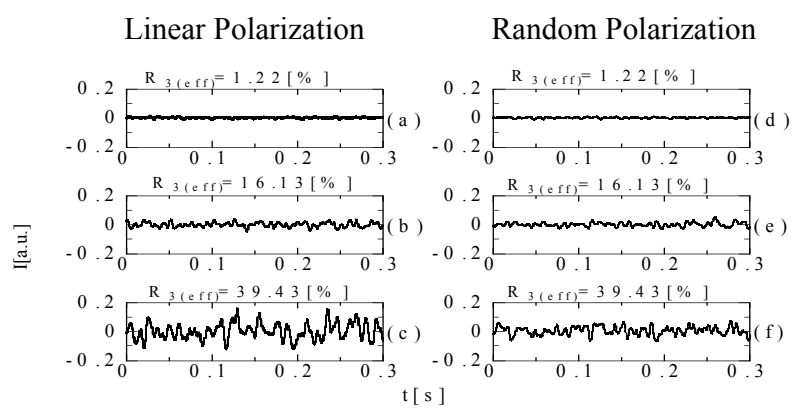

Fig.2. Experimental results of output power(I) from the external mirror, in which the length of the external resonator is $12.3 \mathrm{~cm}$. In the case of linear polarization, from top to bottom: $\mathrm{R}_{(3 \mathrm{eff})}$ is (a) $1.22 \%$, (b) $16.13 \%$,(c) $39.43 \%$. In the case of random polarization, from top to bottom: $\mathrm{R}_{(3 \mathrm{eff})}$ is (d) $1.22 \%$, (e) $16.13 \%$,(f) $39.43 \%$.

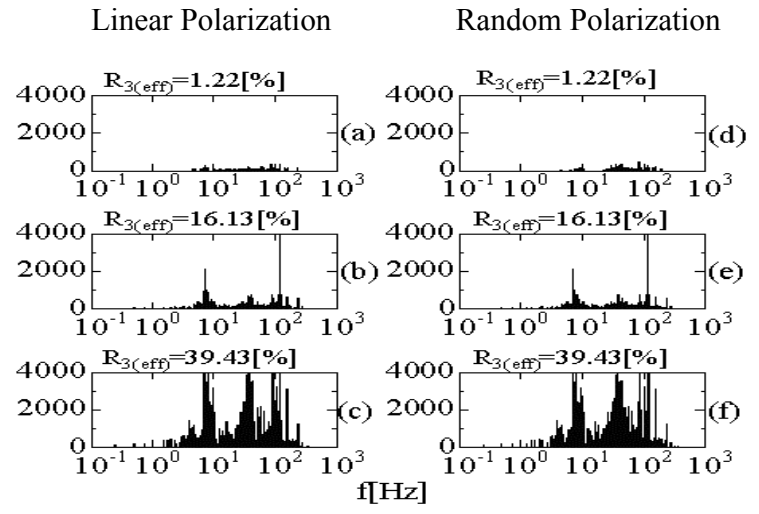

Fig.3. Spectra for experimental results in Fig.2. In the case of linear polarization, from top to bottom: $\mathrm{R}_{\text {(3eff) }}$ is (a) $1.22 \%$, (b) $16.13 \%$, (c) $39.43 \%$. In the case of random polarization, from top to bottom: $\mathrm{R}_{\text {(3eff) }}$ is (d) $1.22 \%$, (e) $16.13 \%$, (f) $39.43 \%$.

internal cavity. Feedback rates are adjusted by the ND filter. The polarization in Figs. 2(a)-2(c) is linear and that of in Figs. 2(d)-2(f) is random. The zero level in each figure corresponds to the steady- state level of $\mathrm{CW}$ operation, and only the variations of output power are shown.

In the case of linear polarization, the $\mathrm{CW}$ steady-state behaviour appears in Fig.2(a). As the feedback rate increases, the output power fluctuates, as shown in Fig.2(b). When the $\mathrm{R}_{(3 \mathrm{eff})}$ is greater than $15.4 \%$, chaotic oscillation occurs and a typical time sequence at $\mathrm{R}_{(3 \mathrm{eff})}=39.4 \%$ above the chaos threshold value is shown in Fig.2(c).

Also in the case of random polarization, the $\mathrm{CW}$ steady-state behaviour appears in Fig.2(d). As the feedback rate increases, the output power fluctuates, as shown in Fig.2(e). When the $\mathrm{R}_{(3 \mathrm{eff})}$ is greater than $21.2 \%$, chaotic oscillation occurs and a typical time sequence at $\mathrm{R}_{(3 \mathrm{eff})}=39.4 \%$ above the chaos

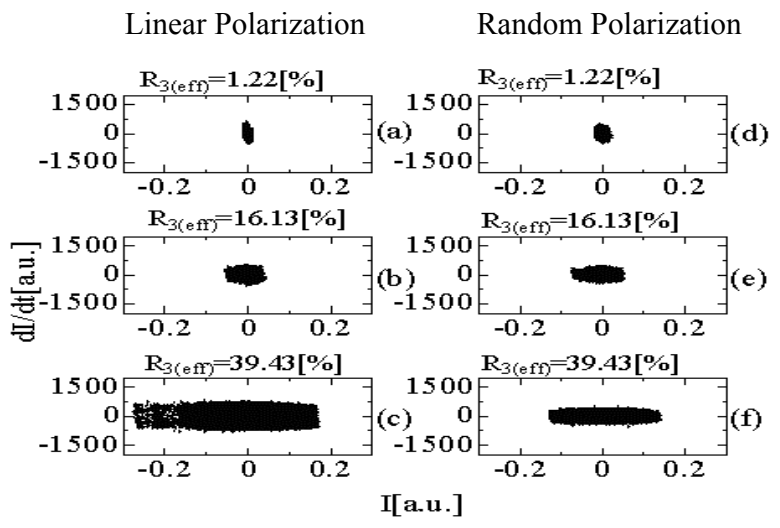

Fig.4. Attractors for experimental results in Fig.2. In the case of linear polarization, from top to bottom: $\mathrm{R}_{(3 \mathrm{eff})}$ is (a) $1.22 \%$, (b) $16.13 \%$, (c) $39.43 \%$. In the case of random polarization, from top to bottom: $\mathrm{R}_{(3 \mathrm{eff})}$ is (d) $1.22 \%$, (e) $16.13 \%$, (f) $39.43 \%$. 
threshold value is shown in Fig.2(f).

The fluctuation for the linear polarization laser is greater than that for the random polarization laser. It shows that the effect of the light polarization on the fluctuation of output power is remarkable.

The spectra are shown in Fig.3(a)-(f), which corresponds to each time series in Fig.2. Under the chaos threshold, there are some peaks from 10 to $100 \mathrm{~Hz}$. As the feedback rate increase, the output power fluctuates and spectrum is broadened as is shown in Fig.3(b) and Fig.3(e). In the case of $\mathrm{R}_{(3 \mathrm{eff})}=39.4 \%$, the spectrum is broadened at approximately $10-100 \mathrm{~Hz}$, resulting chaotic oscillations. These spectra are characteristic components of the chaotic oscillation in this single-mode class A laser (He-Ne,6328 A).

The spectra of linear polarization laser and that of random polarization laser are similar.

This suggests that the polarization effect on the population inversion is so small that can be ignored.

Phase portraits are shown in Fig.4, which corresponds to each time series in Fig.2.

For the case of linear polarization laser, a fixed point appears, which corresponds to a $\mathrm{CW}$ steady state in the case of $\mathrm{R}_{(3 \mathrm{eff})}=1.22 \%$ [Fig.4(a)]. As the feedback rate increase, the output power fluctuates and area of phase portrait is broadened as is shown in Fig.4(b). In the case of $\mathrm{R}_{(3 \mathrm{eff})}=39.4 \%$, the strange attractor which corresponds to a chaotic oscillation is appeared[Fig.4(c)].

Also for the case of linear polarization laser, a fixed point appears, which corresponds to a CW steady state in the case of $\mathrm{R}_{(3 \mathrm{eff})}=1.22 \%$ [Fig.4(d)]. As the feedback rate increases, the output power fluctuates and area of phase portrait is broadened as is shown in Fig.4(e). In the case of $\mathrm{R}_{(3 \mathrm{eff})}=39.4 \%$, the strange attractor which corresponds to a chaotic oscillation is appeared[Fig.4(f)]. The area of phase portrait of linear polarized laser is larger (about 1.8 times) than that of random polarized laser. It is shown that the light polarization changes the area of the strange attractor in holding similar form.

The most important measure for deterministic chaos is the Lyapunov exponent ${ }^{14)}$. Chaos threshold is estimated in terms of the effective reflectivity of the external mirror $\left(R_{(3 \mathrm{eff})}=T^{2} R_{3}\right)$, where $\mathrm{T}$ is the transparent coefficient of the ND filter.

For a linear and random polarization laser, chaos threshold is obtained at $\mathrm{R}_{(3 \mathrm{eff})}=15.4 \%$ and $21.2 \%$, respectively . The ratio of threshold value of random polarization laser to that of linear polarization laser is 1.37 which is similar to $\sqrt{2}$ (the ratio of amplitude to effective value). This effect is notable and also in addition, shown in Fig. 2 even in the same feedback rate, the fluctuation of output of the linear polarization laser is remarkably larger than that of random polarized laser. Furthermore, as mentioned on the spectra the polarization effect on the population inversion is small enough to ignore. Then it is revealed that the delayed feedback light behaves like as directly interacting with the electric field of the inner cavity (see Eq.(23) in ref. 12). Of course, the electric field slaves the population inversion and electric dipole moments that have very faster decay rates than that of the electric field.

\section{Concluding Remarks}

Effects of the light polarization on a chaotic oscillation dynamics of a single-mode $\mathrm{He}-\mathrm{Ne}(6326 \AA)$ class A laser was studied by using optical delayed feedback. The fluctuation of the linear polarization is notably larger than that of random polarized laser and chaos threshold of a linear polarized laser is distinguishably lower than that of random polarized laser. Then the electric field of a delayed feedback light is directly interacted to the electric field not via population inversion. The adiabatic elimination of a population inversion is useful in a class A laser and the electric field takes dominant roll in this class laser.

\section{References}

1) Droller and F. Takens : Commun. Math. Phys. 20 (1971) 167.

2) H. Haken : Phys. Lett. A 53 (1975) 77.

3) C. O. Weiss and H. King : Opt. Commun. 44 (1982) 59.

4) N. B. Abraham, P. Mandel and L. M. Narducci : Dynamical instabilities \& Pulsations in Lasers (Elsevier Science Publishers, B.V.,1988).

5) C. O. Weiss and R. Vilaseca : Dynamics of lasers (Weinheim, New York, Basel, Cambridge, 1991).

6) F. T. Arecchi, G. L. Lippi, G. P. Puccioni and Tredicce : Opt. Commun. 51 (1984) 308.

7) V.I. Chetverikov : Sov. Tech. Phy. Lett. 11 (1985) 190.

8) M.V. Danileiko, A.L. Kravchuk, V. N. echiporenko, A.M. Tselinko and L.P. Yatsenko : Sov. J. Quantum Electron. 16 (1986) 1420.

9) F.Kuwashima, I.Kitazima and H.Iwasawa :Rev. Las. Eng. .24 (1996) 363 [in Japanese].

10) F.Kuwashima, .I.Kitazima and H.Iwasawa : Jpn. J. Appl. Phys. 37 (1998) L325.

11) F.Kuwashima, T.Ichikawa, I.Kitazima and H.Iwasawa : Jpn. J. Appl. Phys. 38 (1999) 6321

12) F.Kuwashima, I.Kitazima and H.Iwasawa: Jpn. J. Appl. Phys. 40 (2001) 601.

13) F.Kuwashima and H.Iwasawa :Jpn.J.Appl.Phys. 46 (2007)1526.

14) A. Wolf, J. B. Swift, H.L. Swineey and J. A. Vastano : Physica D $16(1985) 285$ 